\title{
Chaotic Advection in an Archipelago*
}

\author{
IRINA I. RYPINA AND LAWRENCE J. PRATT \\ Woods Hole Oceanographic Institution, Woods Hole, Massachusetts \\ JULIE PULLEN \\ Stevens Institute of Technology, Hoboken, New Jersey \\ JULIA LEVIN \\ Institute of Marine and Coastal Sciences, Rutgers University, New Brunswick, New Jersey \\ ARNOLD L. GORDON \\ Lamont-Doherty Earth Observatory, Columbia University, Palisades, New York
}

(Manuscript received 18 August 2009, in final form 22 March 2010)

\begin{abstract}
Techniques from dynamical systems theory have been applied to study horizontal stirring of fluid in the Philippine Archipelago. The authors' analysis is based on velocity fields produced by two high-resolution ( 3 and $6 \mathrm{~km}$ ) numerical models. Particular attention is paid to identifying robust surface flow patterns and associating them with dominant Lagrangian coherent structures (LCSs). A recurrent wind-driven dipole in the lee of the coastline is considered in detail. The associated LCSs form a template for stirring, exchange, and biological transport in and around the dipole. Chaotic advection is argued to provide a relevant framework for interpreting mesoscale horizontal stirring processes in an archipelago as a whole. Implications for the formation of filaments, the production of tracer variance, and the scale at which stirring leads to mixing are discussed in connection with an observed temperature record.
\end{abstract}

\section{Introduction}

Mixing in the ocean is generally defined as an irreversible property exchange between neighboring water masses. Mixing can be catalyzed by stirring, a conservative process in which fluid elements are stretched and folded, thereby amplifying property gradients. At some point, the gradients become sufficiently large that smallscale, irreversible processes, often modeled as diffusion, intervene. Strictly speaking, this intervention occurs at the molecular level, but the concept is extended to include all unresolved small-scale processes in ocean

* Lamont-Doherty Earth Observatory Contribution Number 7350.

Corresponding author address: Irina I. Rypina, Physical Oceanography Department, Woods Hole Oceanographic Institution, Woods Hole, MA 02543.

E-mail: irypina@whoi.edu models. The work described herein will concentrate on horizontal stirring on scales ranging from small gyres and mesoscale eddies, roughly $50-200 \mathrm{~km}$ in our case, down to the grid scale of 3-6 km.

Turbulent flows produce rapid stirring and mixing, particularly if fully three-dimensional (3D) vortex stretching is permitted. An isolated patch of dye introduced into 3D turbulence will generally spread rapidly and completely throughout the flow field. However, turbulence is not a prerequisite, nor does the flow in question need to be stochastic or exceptionally complex for rapid stirring and mixing to occur. For example, it is known that rapid two-dimensional stirring occurs on both sides of the polar night jet at the edge of the stratospheric polar vortex, where the flow is dominated by long-lived, coherent eddies associated with planetary waves. The velocity fields of the eddies stretch blobs of air on isentropic surfaces into thin, elongated filaments. The generation of small scales can be simulated simply by allowing tracers to be passively advected by a reasonable approximation 
of the large-scale velocity field (Ngan and Shepherd 1999a,b; Mizuta and Yoden 2001; Koh and Plumb 2000; Koh and Legras 2002; Plumb 2002; Rypina et al. 2007). Similar behavior can be found in idealized models of eddies, meandering jets, and wave forms in 2D or quasi2D eddy fields with relatively simple velocity fields (e.g., Rogerson et al. 1999; Samelson 1992; Poje and Haller 1999; Sommeria et al. 1989). These examples do not fit the traditional definitions of turbulence (Tennekes and Lumley 1972, chapter 1); for one thing, the Eulerian velocity fields are deterministic and often time periodic or quasiperiodic. On the other hand, the Lagrangian properties of such flows can be quite complex. Fluid parcels are sequentially stretched into long filaments and then folded, leading to formation of Smale horseshoes and causing rapid (exponential in time) separation between neighboring fluid particle trajectories. ${ }^{1}$ As in the case of the polar vortex, the generation of small scales is controlled by long-lived, coherent structures, and the stirring process is therefore referred to as chaotic advection. By "long lived," one generally assumes that the coherent structures remain intact over a time period long in comparison with the typical parcel winding time.

It is not uncommon for flows undergoing chaotic advection to contain "barriers" consisting of material contours that are not in a state of Lagrangian chaos. Their existence is predicted by the Kolmogorov-ArnoldMoser (KAM) theorem. The barriers sometimes enclose islands of fluid in which trajectories separate at a rate linear in time and are therefore nonchaotic. Islands of this type often exist in the core regions of the coherent structures.

In the ocean, motion over the scales mentioned earlier is approximately $2 \mathrm{D}$, either on the free surface or along an isopycnal surface. Within the realm of $2 \mathrm{D}$ or quasi2D flows, there are some that exhibit characteristics of chaotic advection and some that more closely resemble classical turbulence. The matter rests on the manner of forcing, the form of the dissipation, and the configuration of the boundaries. One rule of thumb (e.g., Lukovich and Shepherd 2005; Shepherd et al. 2000; Bennett 1984) involves the rate of decay of the kinetic energy density $E(k)$ in horizontal wavenumber $(k)$ space. Suppose that $E(k) \propto k^{-n}$, with $n>3$, over a certain range of the spectrum. Then it can be argued that, over this range of $k$, vorticity at the smaller scales of the range is simply advected as a passive tracer by the large-scale eddies or gyres. Stirring at small scales is controlled by the large

\footnotetext{
${ }^{1}$ Note, however, that exponential separation alone does not guarantee chaotic behavior; it is the Smale horseshoe stretchingand-folding mechanism that causes efficient stirring.
}

scales, and chaotic advection is appropriate. If $n<3$, the small-scale vorticity is no longer passively advected and stirring responds to and may be dominated by smallscale processes. In such flows, filaments generated by large-scale motions rapidly become distorted and fuzzy. Parameterizations of stirring and mixing ought to be different for chaotic advection and for classical turbulence, with chaotic advection having the advantage of depending only on the scales of the large-scale eddies.

Dynamical systems theory has provided a novel and useful way of analyzing flows in a state of chaotic advection (Samelson and Wiggins 2006). A typical analysis provides a kind of Lagrangian template in which particle pathways, barriers, stretching and folding, and certain transport processes can be visualized. This analysis also suggests ways in which transport or exchanges can be quantified. The same methodology may be applied to 2D turbulence with $n<3$, though interpretation of the results and quantification of transport processes is likely to be most transparent in the case of chaotic advection.

It is natural to ask whether there are regions of the ocean, or scale ranges, over which chaotic advection is a relevant paradigm for horizontal stirring. The submesoscale kinetic energy spectrum at the ocean surface (or along isopycnal surfaces) is not currently known well enough to determine whether $n>3$. Instead, one can look for regions with other favorable features, such as long-lived, coherent structures. Archipelagos would seem to offer a likely setting because the natural basins, islands, and complex shorelines ought to give rise to long-lived eddies and gyres. This work concentrates on the Philippine Archipelago (Fig. 1), the subject of a recent field and modeling program [Philippines Experiment (PHILEX)]. The focus is on the surface (or near-surface) flow field, and we rely on high-resolution numerical models to provide surface velocities. On the basis of these model results, we will argue that chaotic advection is a reasonable paradigm for stirring over the archipelago as a whole, from the $50-200-\mathrm{km}$ scale of small gyres and eddies down to $3-6 \mathrm{~km}$. We will also present a detailed analysis of a particular recurrent coherent feature: a dipole generated by a wind jet in the lee of a coastline. The analysis will illustrate a process by which surface water is stripped away from the lee coastline, swept through the axis of the dipole, and stirred around the edges of the eddies in a way that resembles chaotic advection. We will explore the filamentation that occurs as part of this process and estimate the filament width scale at which stirring leads to mixing. We will briefly consider some biological implications.

Our analysis of these fields is Lagrangian in nature and centers on the generation of stable and unstable manifolds (section 2). These objects form the previously 


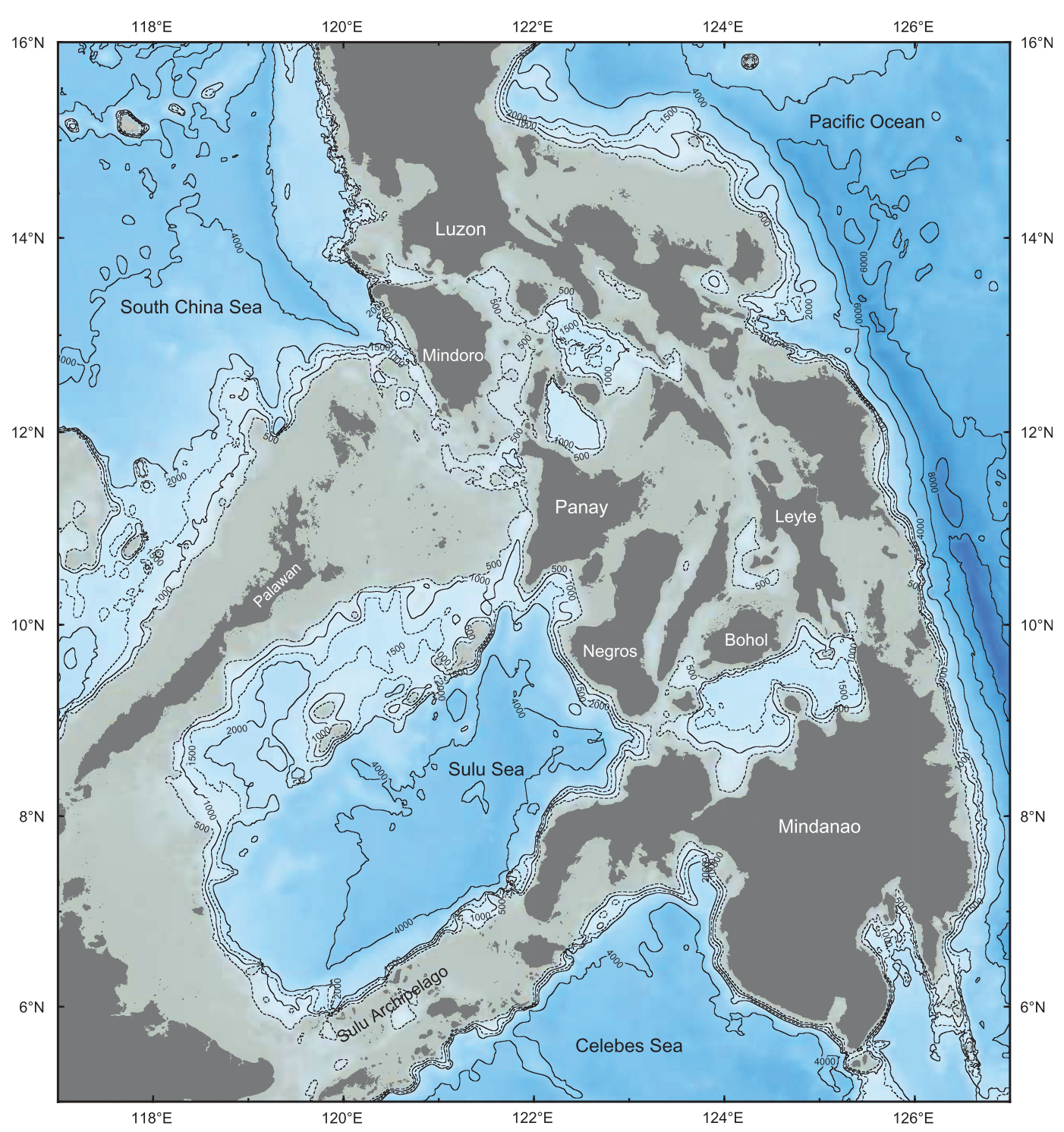

FIG. 1. Philippine Archipelago (from Smith and Sandwell 1997).

mentioned template for the stirring and transport of fluid. The fact that one is able to construct such a picture and show that it is robust and shares elements with known examples is, in itself, evidence that chaotic advection is relevant. A number of methods exist for generating manifolds from numerical data; section 3 describes two standard methods along with a new approach that was tested as part of this work. Section 4 discusses the results of the analysis over the whole archipelago and attempts to make the case that chaotic advection is relevant. Section 5 concentrates on the dipole and associated transport and filamentation processes. The implications for the transport of biological properties are discussed. A summary of our studies and conclusions are given in section 6 .

\section{Review of methodology}

The kinematic origins and geometry of chaotic stirring can be analyzed using theory and numerical methods that have been developed within the dynamical systems community. Most of the foundation is described in the monograph of Samelson and Wiggins (2006) and in a number of papers (Wiggins 2005; Mancho et al. 2006; Haller 2001a,b, 2005; Shadden et al. 2005; Rypina et al. 2007, 2009; Miller et al. 1997, 2002; Kuznetsov et al. 2002). Our brief review is nonrigorous and is limited to a simple example that has some connections with a realistic flow analyzed later. Consider a hypothetical dipole structure (Fig. 2a), with a coastline to the east and an eddy field far to the west. Assume that flow is two 


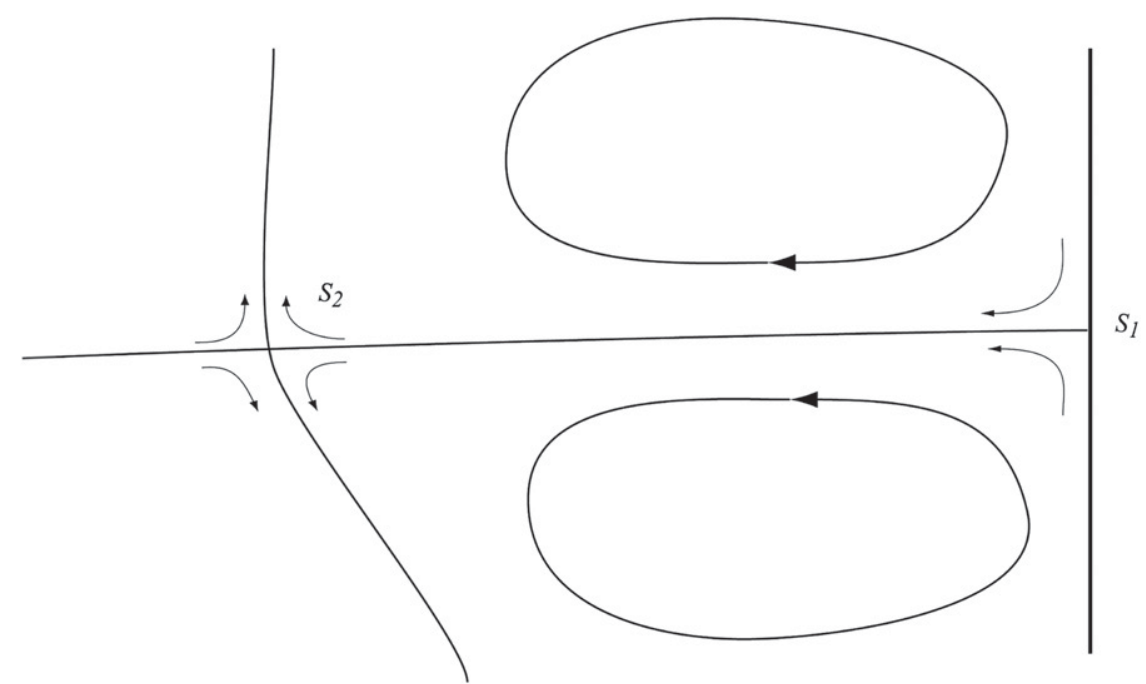

(a)

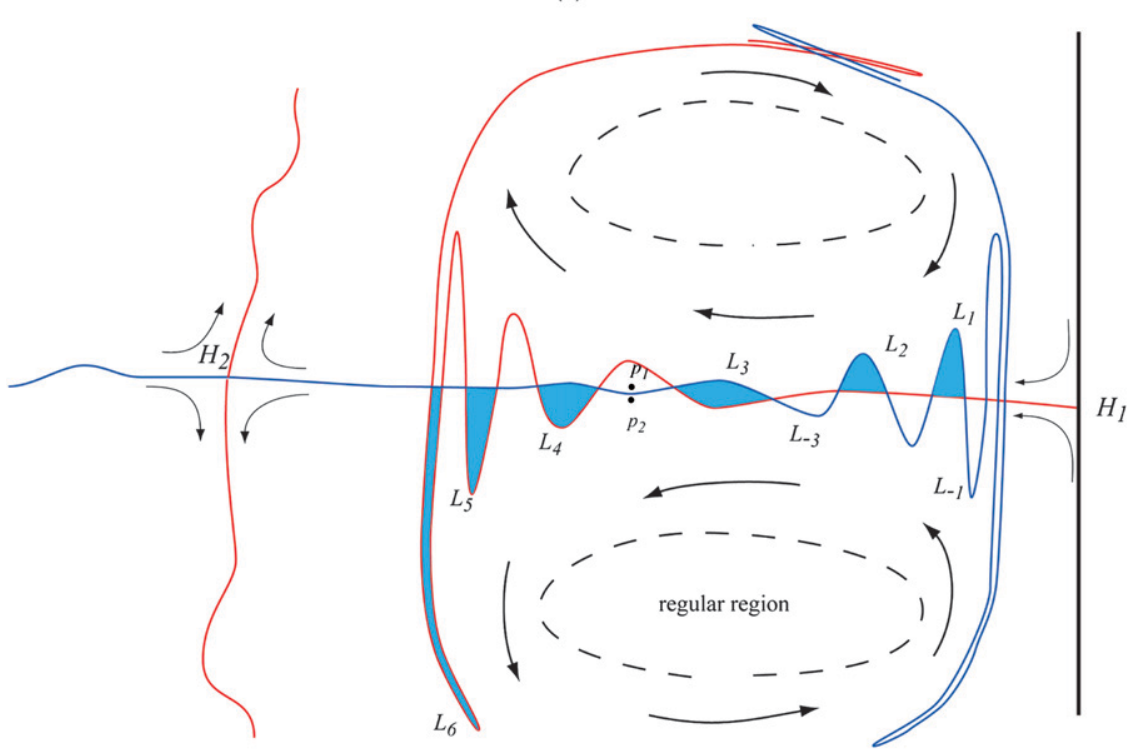

(b)

FIG. 2. (a) Definition sketch showing the streamlines of a hypothetical steady flow containing a cyclonic-anticyclonic eddy pair near an eastern boundary, with an eddy field offshore. (b) The same flow, subject to a weak, time-dependent perturbation.

dimensional and that the velocity field is divergence free. Then a streamfunction $\psi(x, y, t)$ exists and fluid trajectories are described by the Hamiltonian system,

$$
\frac{d x}{d t}=-\frac{\partial \psi}{\partial y} \quad \text { and } \quad \frac{d y}{d t}=\frac{\partial \psi}{\partial x}
$$

If the flow is steady, trajectories and streamlines coincide. The cyclonic and anticyclonic eddies are separated by a streamline that begins at a stagnation point $S_{1}$ on the coast and ends at an internal stagnation point $S_{2}$ to the west of the dipole. Fluid is allowed to slip along the coastline. Both stagnation points are hyperbolic (saddle type), meaning that the surrounding velocity field converges in one direction and diverges in another. (Note that a slightly different geometry, where $S_{1}$ and $S_{2}$ do not lie on the same streamline but rather on two different streamlines, will occur in a later example. The distinction between these two cases is not essential for our discussion, provided that the two streamlines on which $S_{1}$ and $S_{2}$ lie are located close to each other.)

Now suppose that a weak, time-dependent perturbation of the flow field is introduced (Fig. 2b). Then it can 
be shown that generalizations of the objects defined for the steady flow exist. Of these, the most important are hyperbolic trajectories, which are relatives of the steady stagnation points. Loosely speaking, a hyperbolic trajectory corresponds to a distinguishable fluid parcel that experiences the same convergence and divergence of surrounding motion that characterized the earlier stagnation points. In the present context, a hyperbolic trajectory $H_{1}$ can be expected on the eastern boundary in the vicinity of the former stagnation point $S_{1}$. Its position will generally vary with time, but it can be expected to stay in the same region, provided the perturbation is small. Neighboring fluid parcels will converge along the boundary toward $H_{1}$ but veer offshore as the hyperbolic trajectory is approached. If dye is continuously introduced in the vicinity of this trajectory, it will be attracted to $H_{1}$ in a stable direction and then repelled in an unstable direction, forming a thin material contour that will stretch offshore along the unstable direction. This dye line serves as an approximation to the unstable manifold: the material contour consisting of all the trajectories that emanate from the hyperbolic trajectory in forward time (i.e., approach it as $t \rightarrow-\infty$ ). The unstable manifold evolves in time, and its representation in Fig. 2b (the red contour) is just a snapshot.

In a similar manner, the internal stagnation point $S_{2}$ turns into a hyperbolic trajectory $H_{2}$ when a weak timedependent perturbation is introduced. It has (red) unstable manifolds extending roughly to the north and south and (blue) stable manifolds to the east and west. The latter consists of all the material that approaches $H_{2}$ asymptotically in time. Stable manifolds can be mapped out by introducing a passive tracer (dye) in the vicinity of $\mathrm{H}_{2}$ and allowing it to evolve backward in time.

In the steady version of the flow, which is illustrated in Fig. 2a, the unstable manifold of $S_{1}$ is also a stable manifold of $S_{2}$. Each coincides with the streamline that connects $S_{1}$ and $S_{2}$ and separates the cyclonic and anticyclonic parts of the dipole. With the addition of time dependence, this is no longer the case. The unstable manifold of $H_{1}$ executes a series of meanders that become increasingly distended as one moves away from $H_{1}$ to the west. A similar property holds for the stable manifold of $\mathrm{H}_{2}$ as one moves away from $\mathrm{H}_{2}$ to the east. Thus, the manifolds that previously combined to form the steady, separating streamline are now distinct entities. They generally intersect each other and form a pair of tangled curves. In time-periodic or quasiperiodic flows, this tangling indicates that fluid trajectories in the vicinity are chaotic. Chaos is manifested by the generation of Smale horseshoes leading to sequential stretching and folding of fluid parcels and causing rapid (exponential in time) separation of closely spaced fluid elements, as exemplified by two fluid parcels $p_{1}$ and $p_{2}$ initially situated on opposite sides of an unstable manifold (Fig. 2b). This rapid separation occurs when the parcels approach $\mathrm{H}_{2}$, the first drifting north and the other south. A short piece of material connecting the two elements clearly gets stretched at a rapid rate. The timeaveraged stretching rate of an infinitesimal fluid element is quantified by the finite-time Lyapunov exponent.

Rapid separation of closely spaced fluid elements also occurs in the steady case (Fig. 2a) but is limited to fluid elements lying infinitesimally close to the separating streamline. The presence of time dependence extends the fluid affected to finite areas, the extent of which is determined by the strength and character of the perturbations. These areas, often referred to as chaotic regions, are densely covered by the stable and unstable manifolds. However, for sufficiently weak perturbations, there are remaining finite regions in which the Lagrangian motion is nonchaotic (regular). The existence of such regions is predicted by the celebrated KAM theorem, which states that, provided certain conditions are met, some trajectories in the vicinity of certain closed material curves will not become chaotic. In the current example, regular regions can be expected to arise in the central regions of the interiors of the two steady eddies. Neighboring fluid parcels in regular regions separate from each other at a rate that is only algebraic in time.

The tangling of the manifolds governs transport processes by which fluid is exchanged between the predominantly cyclonic and anticyclonic parts of the dipole. Consider the lobe $L_{1}$ of fluid whose edges are defined by segments of the stable and unstable manifolds (Fig. 2b). The manifolds are material contours, and thus the fluid in the lobe remains trapped between the same two segments for all time. The predominant direction of flow is westward at the location shown, and thus the lobe can be expected to move in this direction as time progresses. The shapes of the manifolds evolve during this process, but if the time dependence is periodic then the shapes reemerge periodically. After one period, the fluid in $L_{1}$ may be expected to evolve to $L_{2}$, then to $L_{3}$, etc. A qualitatively similar process can occur even when the time dependence is aperiodic. In either case, fluid from the southern part of the flow field is being transported northward, across the position of the former separating streamline. At the same time, material trapped in the lobes $L_{-1}, L_{-2}$, etc. is being transported from the northern to the southern region. As the lobes evolve, they preserve their area but become increasingly distended and folded. Passive tracers carried by them become stretched and folded into fine filaments. This is the essence of the rapid stirring. Note that the lobes do not 
penetrate into the regular regions in the interior of the eddies.

Although the regimes sketched in Fig. 2b represent a common scenario, it by no means is the only possibility. This is particularly true when the time-dependent perturbation is strong, in which case the regular regions may not exist, additional hyperbolic trajectories can occur, and other complications may arise.

Numerically computed structures that approximate barriers to chaotic transport and stable/unstable manifolds of hyperbolic trajectories are sometimes referred to as Lagrangian coherent structures (LCSs). We have found examples of these objects in the near-surface velocity field of the Philippine Archipelago, as represented by models. The LCSs arise in connection with eddies, gyres, and coastal separation points that exist within the region. These flows are horizontally divergent (though weakly so over most of the area) and are known only over finite time, so two-dimensional hyperbolic trajectories and manifolds do not exist in a formal sense. In such flows, the term "chaos" is used loosely and the distinction between chaotic and regular motion is blurred. (Note that exponential growth over any finite time interval can be approximated with arbitrary precision by a polynomial.) However, it is possible to define the trajectory that a surface drifter, advected by the horizontal surface velocity, would have. From the collection of all such trajectories, it is possible to identify objects that resemble hyperbolic trajectories and to generate "effective" stable and unstable manifolds based on the horizontal velocity. The latter could be interpreted as contours consisting of all the surface drifter trajectories that approach or diverge from the hyperbolic trajectories within a specified time interval. For most practical purposes, these objects play the same role as their more formally defined counterparts.

\section{Analysis techniques}

The Philippine Archipelago is a complex and sparsely observed region of straits, basins, and semienclosed seas. It is forced by winds, tides, boundary inflows and outflows, river runoff, and surface buoyancy fluxes. Observations of velocity fields are generally too sparse to permit calculation of LCSs, so we instead rely on models. Our analysis in this section is based on the 6-hourly averaged near-surface velocities (averaged over the upper $15 \mathrm{~m}$ ) produced by the Regional Ocean Modeling System (ROMS). ROMS is a three-dimensional, freesurface, terrain-following ocean model that solves the Reynolds-averaged Navier-Stokes equations using the hydrostatic vertical momentum balance and Boussinesq approximation (Shchepetkin and McWilliams 2005;
Moore et al. 2004). The Philippine ROMS model currently uses a $6-\mathrm{km}$ horizontal resolution and 42 terrainfollowing vertical levels stretched to give high resolution in a surface boundary layer. Open boundary temperature, salinity, and subtidal frequency velocity are taken from a bias corrected $1 / 12^{\circ}$ Hybrid Coordinate Ocean Model (HYCOM) data assimilation product. Bias correction is based on replacing mean HYCOM temperature and salinity with Levitus 2005 climatology. Tides have been introduced at the boundary using harmonic data from the Oregon State University Ocean Topography Experiment (Topex)/Jason altimetry data inversion (Egbert and Erofeeva 2002) and a surface gravity wave radiation scheme (Flather 1976). Air-sea heat and momentum fluxes are computed using bulk formulae (Fairall et al. 2003) applied to ROMS sea surface fields, and air temperature, pressure, humidity, and winds are obtained from $1 / 2^{\circ}$ U.S. Navy Operational Global Atmospheric Prediction System (NOGAPS) atmospheric model.

Our analysis of near surface velocity began with a search over a broad area for features of interest. As part of the search, we created snapshots of all the stable and unstable manifolds that can reasonably be resolved within the area. Among other things, the resulting maps reveal features of interest and allow one to approximate the associated hyperbolic trajectories, manifolds, and barriers. Some of these features occur at interior locations, but many are generated in the vicinity of flow separation or stagnation along the boundaries of the $\sim 7000$ islands that compose the archipelago.

A common technique (Haller 2002; Shadden et al. 2005) for calculating approximate locations of stable and unstable manifolds is based on the finite-time Lyapunov exponent (FTLE), defined as the maximum exponential separation rate between a trajectory and its close neighbors, averaged over a finite time. ${ }^{2}$ A fluid parcel initially lying close to a stable manifold will experience rapid separation from its neighbors on the other side of the manifold as it approaches and then passes the corresponding hyperbolic trajectory. Thus, fluid parcels in the vicinity of a stable manifold can be expected to have relatively high values of the FTLE computed in forward time (FTLE-f). Similarly, fluid parcels in the vicinity of an unstable manifold are expected to have high values of the FTLE computed in backward time (FTLE-b). With this in mind, stable/unstable manifolds can be

\footnotetext{
${ }^{2}$ Values of FTLEs are estimated through the logarithm of the largest eigenvalue of the Cauchy-Green deformation tensor. Components of this tensor are estimated by taking the difference in $x(t)$ or $y(t)$ between the positions of initially close trajectories.
} 
approximated as maximizing ridges of FTLE-f/FTLE-b (Shadden et al. 2005).

A recently developed alternative technique for calculating approximate positions of stable and unstable manifolds relies on measures of complexity of individual particle trajectories rather than on measures of separation rates between trajectories. We refer to these methods as individual trajectory methods (ITMs). One such complexity measure is the trajectory correlation dimension (CD), closely related to the fractal dimension. The basic principles of the ITMs are explained in appendix B.

\section{Overview of Lagrangian coherent structures in the archipelago}

We first consider a broad view of LCSs over the whole archipelago by presenting a snapshot of the stable and unstable manifolds on 3 March 2009 (Fig. 3). The shading in Fig. 3a corresponds to FTLE-f values calculated over the 7-day period beginning on that date. The largest values occur as darkened contours or ridges, and these approximate stable manifolds. Figure $3 \mathrm{~b}$ shows unstable manifolds based on FTLE-b: that is, calculated backward in time from 3 March over a 7-day period. The stable and unstable manifolds are superimposed in Fig. 3c. The pictures are quite complex, but one feature that immediately stands out is that the most robust LCSs over the indicated time period are associated with the mesoscale features (gyres, eddies, etc.) and are not appreciably influenced by submesoscale turbulence (in which case one would see a complicated web of small-scale tangled manifolds that cover the domain). Of course these remarks apply only to submesoscale activity that is actually resolved by the $6-\mathrm{km}$ grid and that has not been smoothed by implicit or explicit diffusion acting at small scales.

One can make out a number of organized structures, including a twin-eddy structure in the western Sulu Sea (labeled 1 in Fig. 3c). Stirring associated with this feature is governed by manifolds that emanate from several internal hyperbolic trajectories, one of which can be found near $7.5^{\circ} \mathrm{N}, 118.5^{\circ} \mathrm{E}$. Another area of interest occurs in the south, just to the north of the Sulu Archipelago. Exchange with the Celebes Sea occurs here, and one can see a complicated set of manifolds that arc over the region to the north of the passage. Another interesting feature is a boundary-trapped eddy in the southern Mindanao Sea (labeled 2). When present, this feature may have a large influence on the Pacific to Sulu Sea Throughflow. Other features of interest include a coastal gyre near the entrance to San Bernardino Strait (labeled 3) and a number of eddies or gyres along the western coastline of Negros Island.
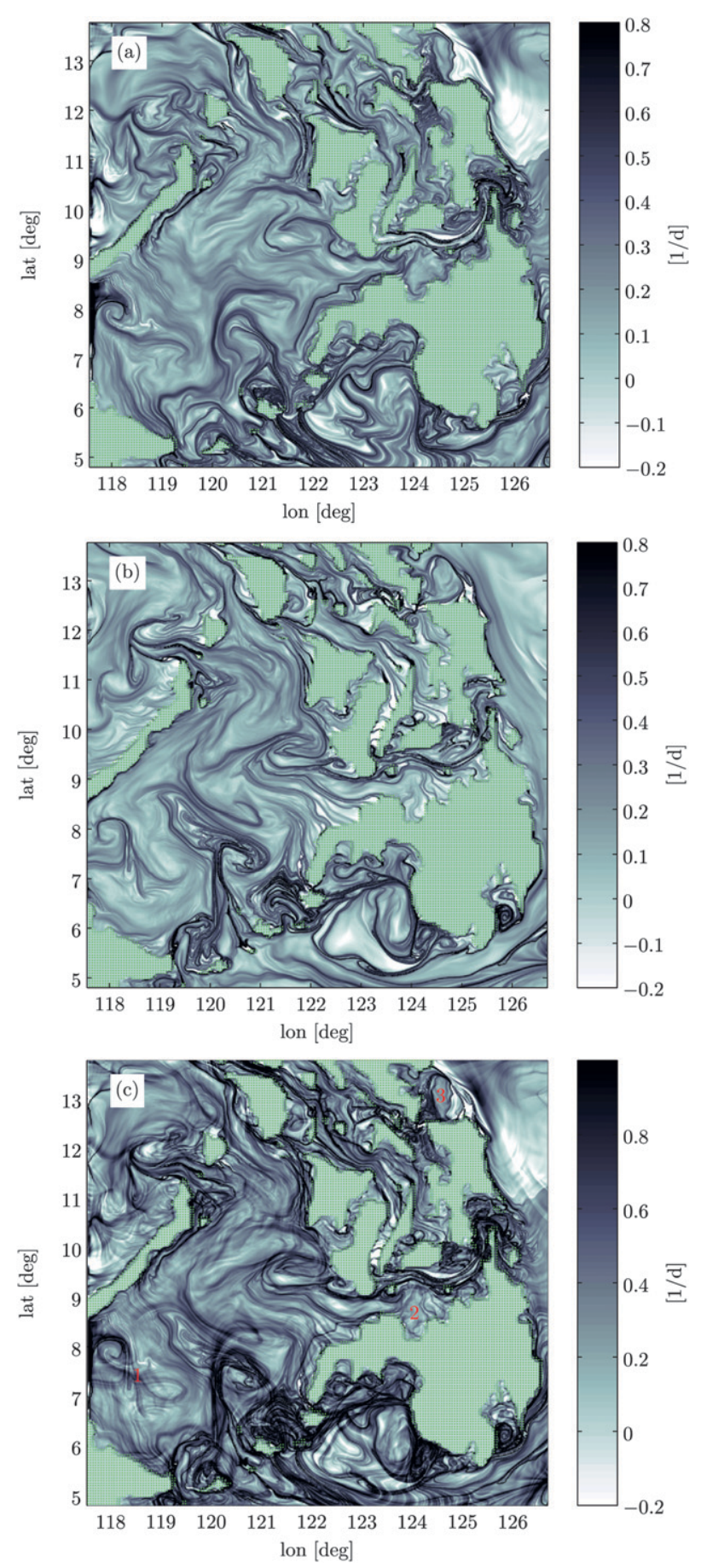

FIG. 3. (a) FTLE-f calculated in forward time for trajectories initiated on 3 Mar 2009 and calculated over a 7-day span. The trajectories are calculated from ROMS model near-surface, horizontal velocities. The maximizing ridges approximate stable manifolds for the near-surface horizontal flow. (b) Unstable manifold for the same date based on the FTLE-b. (c) Superposition of manifolds from (a),(b). 


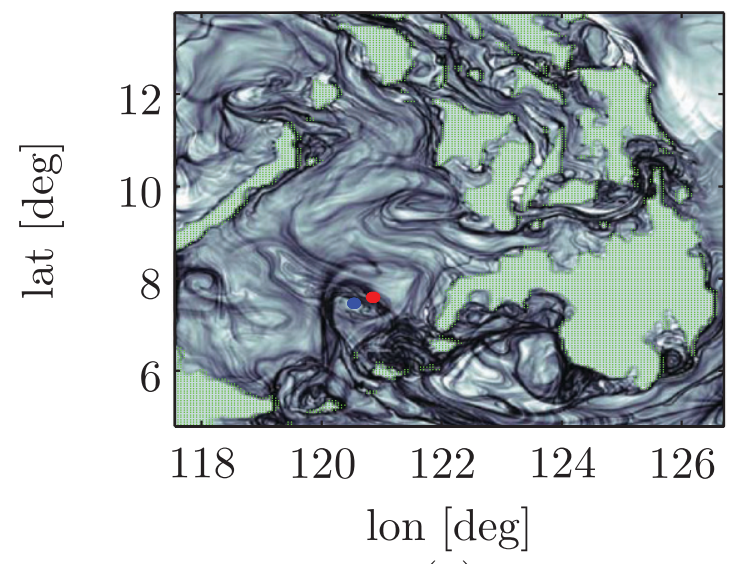

(a)

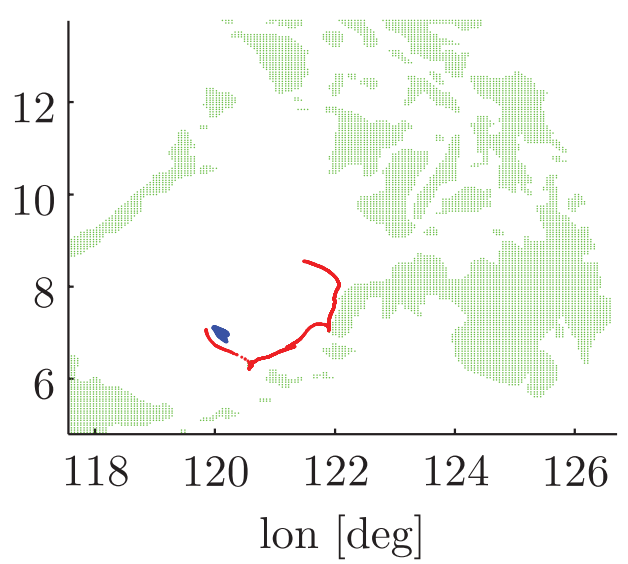

(b)

FIG. 4. Evolution of two clusters of surface drifters launched from a region to the immediate north of the Sibutu Passage. (a) The red cluster is placed over a region of tangled manifolds, whereas the blue cluster is placed in a region free of manifolds. (b) The drifter patches seven days later.

In textbook examples of chaotic advection, the flow field is separated into distinct regions of chaotic and nonchaotic motion, the former occupied by tangled manifolds and the latter devoid of manifolds. In the more realistic ocean model shown in Fig. 3, this distinction is less clear cut. Nevertheless, one can identify, by dark, tangled manifolds, regions of relatively rapid stirring and distinguish nearby regions of lighter shading, indicating much weaker stirring. One example is the previously mentioned area slightly to the north of the Sulu Archipelago, which contains some of the highest FTLE values in the entire region. Consider two clusters of surface drifters, one positioned over a region of tangled manifolds (red patch in Fig. 4a) and the other in a nearby region relatively free of manifolds (blue patch). After 7 days, the red patch has become stretched over a large length, whereas the blue patch remains intact (Fig. 4b).

Where are the hyperbolic trajectories that give rise to the manifolds shown in Figs. 3 and 4? The most obvious examples occur along coastlines or islands where flow separates or "stagnates." There is a prominent example of separation of northward flow from the small island located $\left(5.6^{\circ} \mathrm{N}, 120.9^{\circ} \mathrm{E}\right)$ in the Sulu Archipelago. The dark contour that extends northward from the island (Fig. 3b) is an unstable manifold; the dark contour that extends southward from the island (Fig. 3a) is a stable manifold. A fascinating but problematic feature of archipelagos is that hyperbolic trajectories will exist on the boundary of any island, no matter how small, if flow streams past the island. Internal hyperbolic trajectories also exist, but further analysis may be required to distinguish them from ordinary intersections between stable and unstable manifolds. An example is presented next.
For the purpose of analyzing individual structures within the broader flow patterns, it is sometimes desirable to construct manifolds with more precision. If the approximate position of a hyperbolic trajectory is known, the corresponding manifolds can be grown using the so-called direct method: the numerical equivalent to a dye release in forward or backward time (Mancho et al. 2003, 2004, 2006). Direct synthesis of the manifolds also confirms whether indirect (FTLE, CD, etc.) methods are producing correct results. As an example, consider the twin-eddy feature identified as 1 in Fig. $3 \mathrm{c}$ and shown in more detail in Fig. 5. Blue/red curves show segments of the stable/unstable manifolds computed using the direct manifold integration method. Note that the colored curves coincide with ridges of the FTLE field, giving us some confidence that the indirect FTLE method is producing manifolds correctly. The feature consists of two anticyclonic eddies and has a geometry similar to the flow field described by the "Duffing oscillator," often used to construct textbook examples of manifolds and lobe transport. In the steady Duffing oscillator, a single hyperbolic stagnation point exists between two eddies with the same sign of rotation. This stagnation point lies at the intersection of a figure- 8 streamline that encloses the two eddies. In the presence of a weak time-dependent perturbation, the stagnation point becomes a hyperbolic trajectory from which stem stable and unstable manifolds. The perturbed Duffing oscillator flow is characterized by the figure-8-shaped chaotic region (which is densely covered by intersecting stable and unstable manifolds) centered at the hyperbolic trajectory between the gyres and by two regular regions (where no manifolds are present) associated with the centers of the two gyres. The Fig. 5 feature bears qualitative resemblance to this form, 


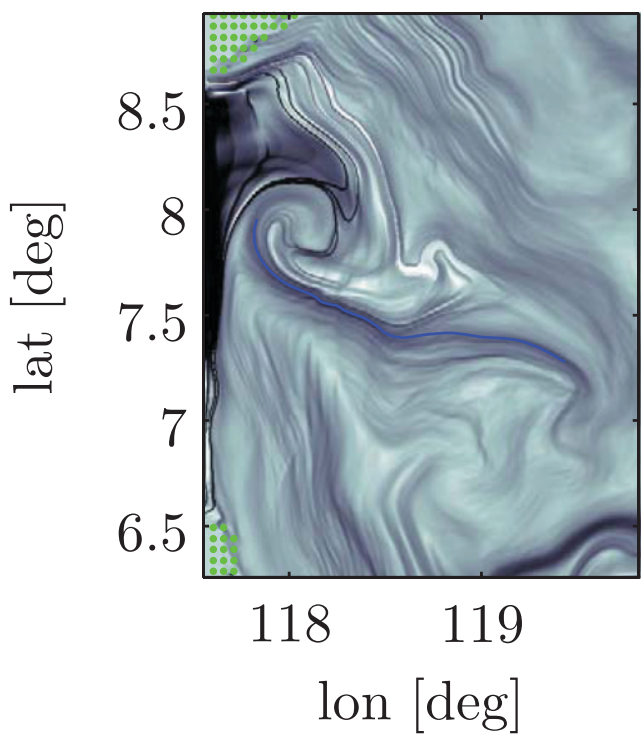

(a)
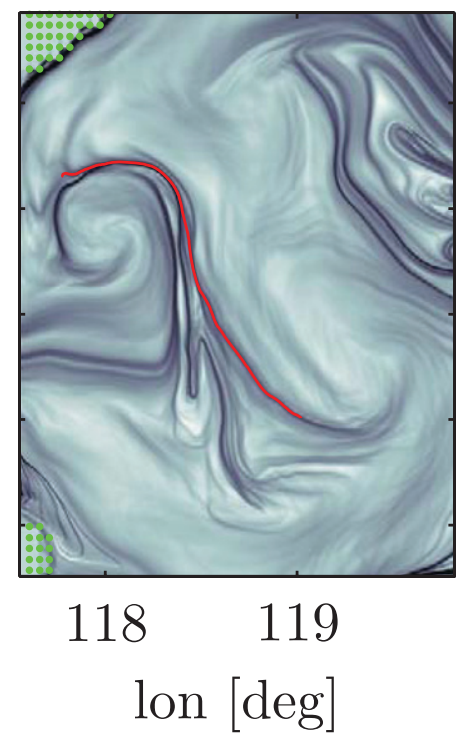

(b)
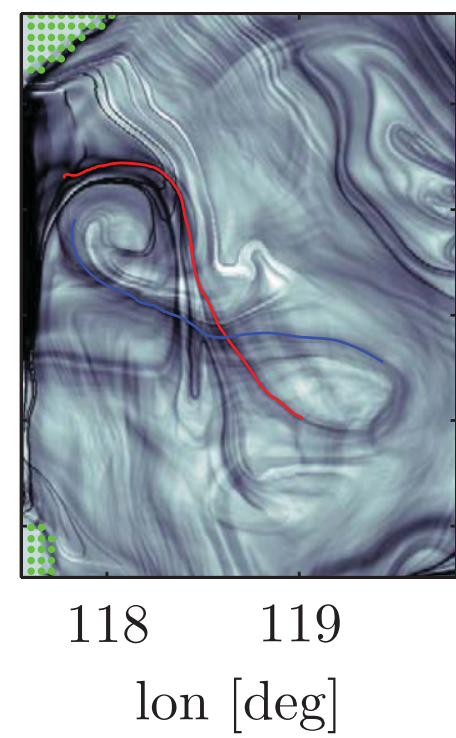

(c)

FIG. 5. The (a) blue and (b) red curves are stable and unstable manifolds grown using a direct manifold evolution method by releasing particles in the vicinity of a hyperbolic trajectory located between two eddies in the western Sulu Sea. The background shading indicates the FTLE-b and FTLE-f values. (c) The results are superimposed.

but there seems to be more than one internal hyperbolic trajectory near the center of the figure 8 from which manifolds emanate. Manifolds are seen to wrap around the centers of the two gyres (where no robust manifolds are present) and form the figure 8. This implies that stirring is enhanced around the perimeter of the two gyres and inhibited near the centers of the gyres.

In summary, a number of coherent circulation features are apparent in the model surface velocity field. Each has a scale at or above the 100-200-km Rossby radius of deformation for the Philippine Archipelago. It is possible to identify within the coherent features objects resembling hyperbolic trajectories and their stable and unstable manifolds. The most robust manifolds produced over time scales of 1 week are associated with mesoscale features, suggesting that the stirring over these time scales is also controlled by the mesoscale structures rather than turbulence at smaller scales. The regions of strong stirring occur in close proximity to regions of weak stirring. Chaotic advection therefore appears to be a relevant concept, as do the tools of analysis described earlier. In the next section, we map out the manifolds of a particular feature (a dipole) in a more precise way and use the results to make deductions about stirring, mixing, and transport.

\section{The VIP dipole}

During the winter monsoon, the northeasterly winds blowing across the archipelago become accelerated in the gaps between islands and between coastal mountains. Strong wind jets are produced in the lee of Mindoro, Panay, and other west-facing coasts. As documented by Pullen et al. (2008, hereafter P08), fluctuations in the jets caused by monsoon surges induce the spinup of coastal ocean dipoles and other eddy structures. P08 documented two dipole occurrences, both generated by the wind stress curl from a westward wind jet in the vicinity of the Verde Island Passage (VIP) and reaching wind speeds in excess of $15 \mathrm{~m} \mathrm{~s}^{-1}$. The dipoles were observed in satellite imagery of the area and were reproduced in model runs using the NCOM ocean model with $3-\mathrm{km}$ horizontal resolution, forced by $8-\mathrm{km}$ Coupled Ocean-Atmosphere Mesoscale Prediction System (COAMPS) model winds (additional model details can be found in P08). The first dipole was generated in late December 2004 and remained near the coast until about 8 January, when it began to move westward. For the next week or so, it moved several hundred kilometers westward, eventually merging into the offshore eddy field. The model-generated surface velocity field for this feature is shown in Fig. 6 for 3 January. With its anticyclonic half to the north and cyclonic half to the south, the dipole's self-propagation tendency is to the west, in agreement with what is observed after the feature breaks away from the coast. The velocity extends down 100-200 $\mathrm{m}$ in the water column, the approximate thermocline depth. A second dipole was generated later in January by a similar wind event, but we will focus on the first case. 


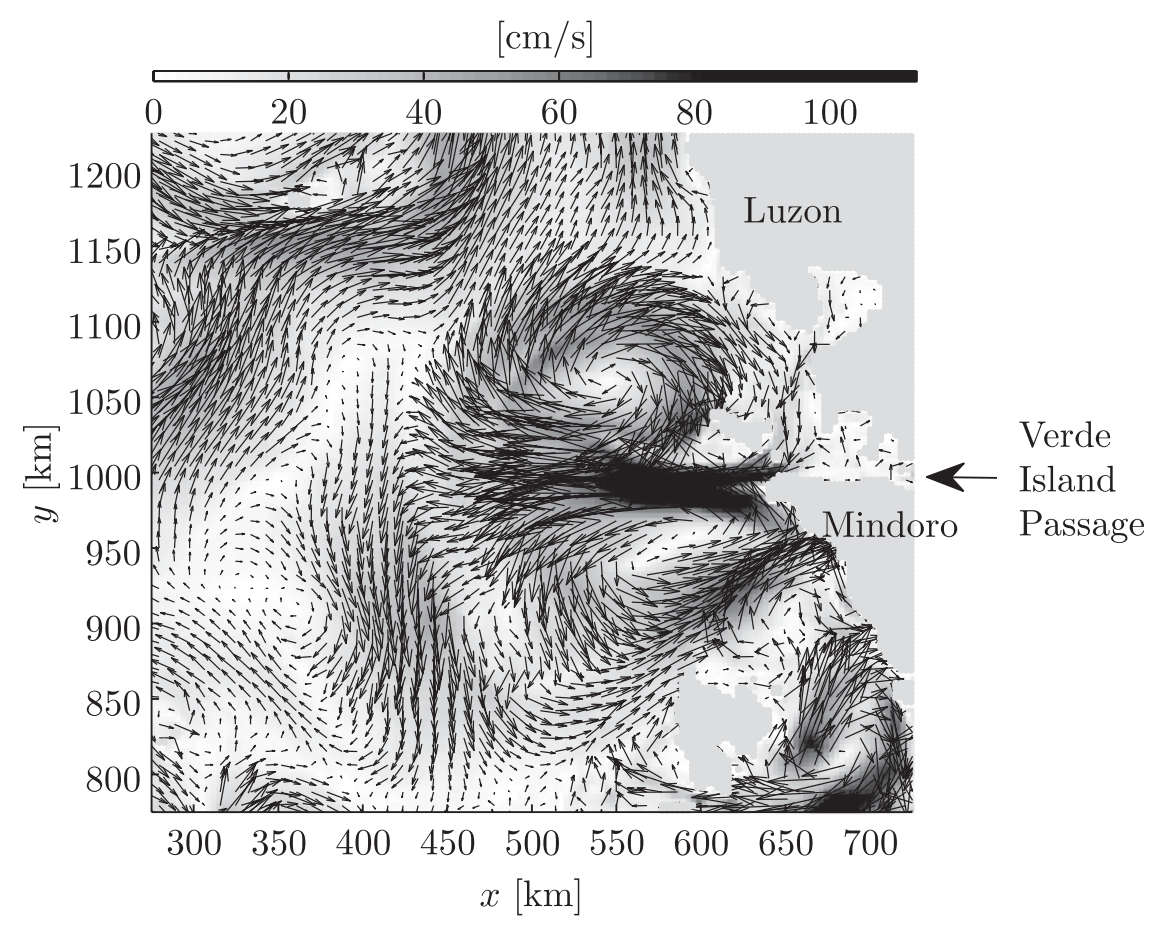

FIG. 6. NCOM model surface velocity to the west of VIP, on 3 Jan 2005.

\section{a. Manifold structures}

The stable and unstable manifolds for the dipole have been calculated using the CD-based method and the direct manifold evolution method. A snapshot of the manifolds for 3 January based on calculations extending 7 days backward and forward in time about this date is shown in Fig. 7. The top panels show the CD-f and $\mathrm{CD}-\mathrm{b}$ fields, and the bottom panel shows manifolds grown using the direct method. Stable/unstable manifolds correspond to minimizing ridges (dark curves) and curves of highest gradient (curves of abrupt color change) in the top left/right panels and to blue/red curves in the bottom panel. Note that the two methods are in good agreement with each other.

Although the overall geometry in Fig. 7 is similar to the cartoon example of Fig. 2b, a comparison between the two produces a good demonstration of the difference between textbook examples and more realistic oceanic flows. Whereas the dipole in Fig. $2 \mathrm{~b}$ contains one stable and one unstable manifold, the Fig. 7 dipole contains several manifolds of each type. The hyperbolic trajectories for the unstable manifolds are associated with separation of westward flow as it passes various peninsulas and islands near the western end of VIP. Three distinct red manifolds stream off to the west, each beginning with a more or less straight alignment but later meandering in the characteristic fashion. At some distance downstream, these manifolds become so closely spaced as to be indistinguishable from a single manifold, at least within the resolution of the model. For most practical purposes, we will treat the three manifolds in this region as essentially one. At about the same latitude, a stable manifold enters the domain from the west, intersecting the red curves in several places and continuing to the coastline to the south of VIP. The origin of this blue contour is an internal hyperbolic trajectory lying in the eddy field to the west of the dipole.

The lobes formed by intersections of this blue contour and the red contour (the composite of three unstable manifolds) contain fluid originating near the mouth of VIP. The lobes move westward from VIP, passing between the cyclone and anticyclone, eventually becoming wrapped around the edges of the two eddies. Fluid transported by the lobes is stretched into thin filaments in the process. The large shaded lobe $L$ in Fig. 7, which resembles qualitatively the lobe $L_{5}$ in Fig. $2 \mathrm{~b}$, can be traced backward in time and can be shown to originate from a lobe generally resembling $L_{1}$ in Fig. 2b. This evolution is demonstrated by a sequence of realizations of $L$ (Fig. 8). On 29 December, the lobe lies near the islands that guard the entrance to VIP. The lobe moves to the west (30 and 31 December) as a rounded blob whose boundary consists primarily of the stable manifold, with a short segment of unstable manifold (similar to the lobe $L_{1}$ of Fig. 2b). However, as the lobe is swept 

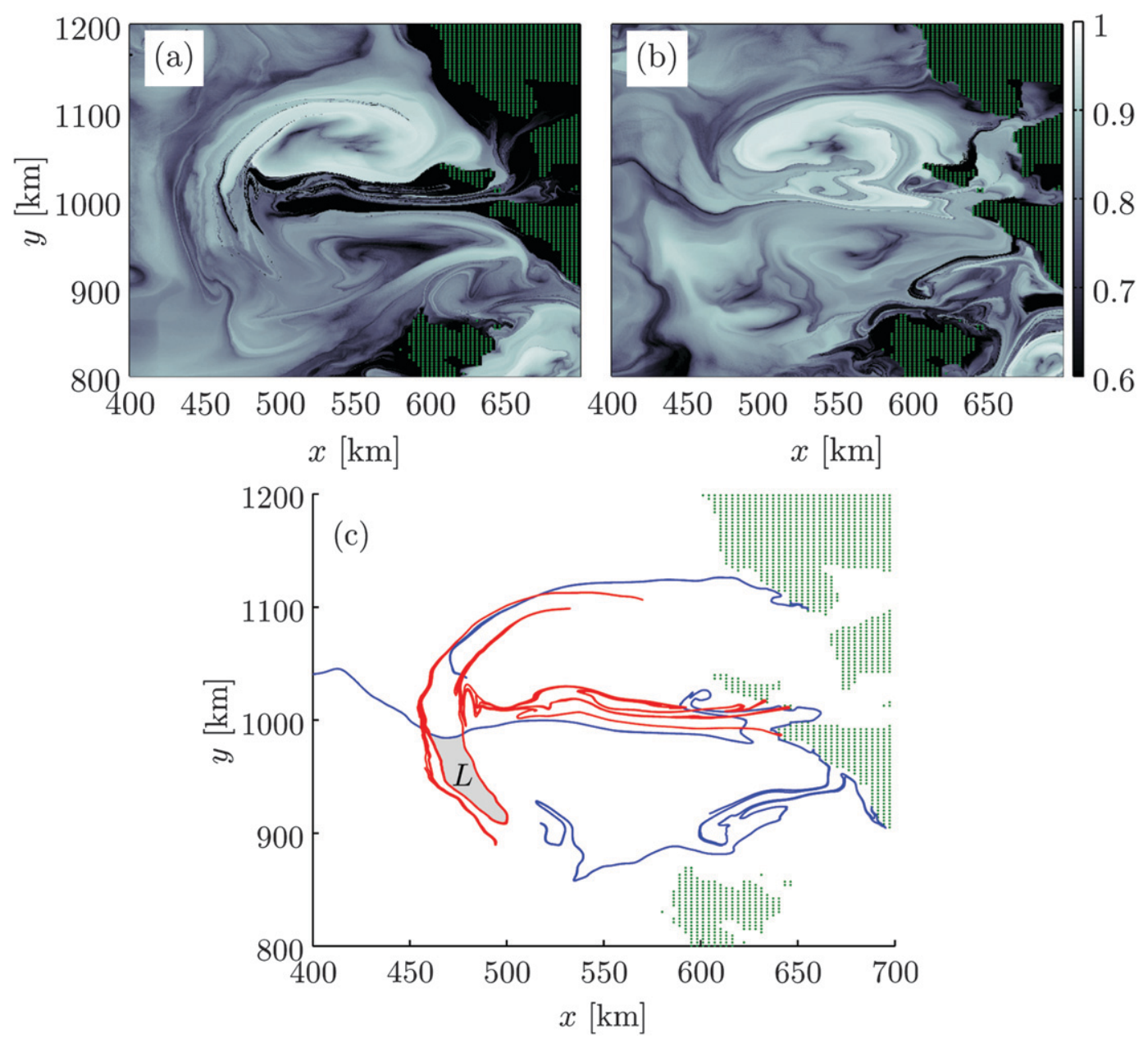

FIG. 7. Stable and unstable manifolds for the VIP dipole as they appear on 3 Jan 2005: (a) CD-b and (b) CD-f. The darkened ridges and curves of abrupt color change approximate stable and unstable manifolds. (c) Stable and unstable manifolds grown using a direct manifold evolution method.

southwestward (1-5 January) it becomes increasingly stretched, and its boundary consists primarily of the unstable manifold (similar to the lobe $L_{6}$ of Fig. 2 b).

The surface area of this lobe does not remain constant, as it would in a divergence-free, $2 \mathrm{D}$ velocity field. These changes are due to convergence and divergence of the near-surface velocity field (and also due to small numerical error associated with the computation of the manifolds). The average area is approximately $1200 \mathrm{~km}^{2}$ or, with a depth of $200 \mathrm{~m}$, a volume of about $3 \times 10^{8} \mathrm{~m}$. This volume is transported approximately $200 \mathrm{~km}$ in 5 days. The time scale for the motion of the lobes is set by a combination of complex processes including tides, wind variations, boundary inflow/outflow, variations of the exterior eddy field, etc.

Stirring in and around the dipole can be further demonstrated by releasing concentrated groups of surface drifters in the eddy core regions (blue patches in Fig. 9) and in a region between eddies that is covered by intersecting manifolds (red patch). The stirring of the blue patches is significantly less pronounced than that of the red patch. A clear distinction between regular and chaotic regions does not exist here: the eddy cores are not completely devoid of manifolds and, even if they were, diffusive, subgrid-scale processes would allow transport into the cores. However, there is a quantitative difference between the regions densely covered by intersecting manifolds and core regions. The Lyapunov exponents for the former are typically about $1 \mathrm{day}^{-1}$, whereas values in the eddy cores are an order of magnitude less.

Finally, it is important to note that the second dipole, which was generated by the wind event at the end of January, has similar qualitative features (hyperbolic trajectories, manifold structure, lobes, etc.) to those discussed in this section. This suggests that the results discussed earlier are not limited to the particular dipole 

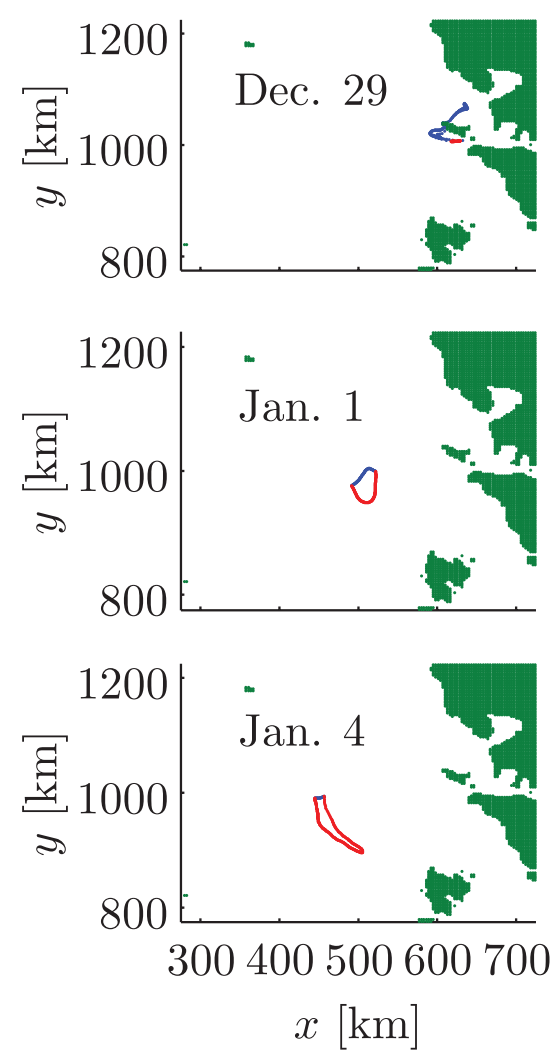
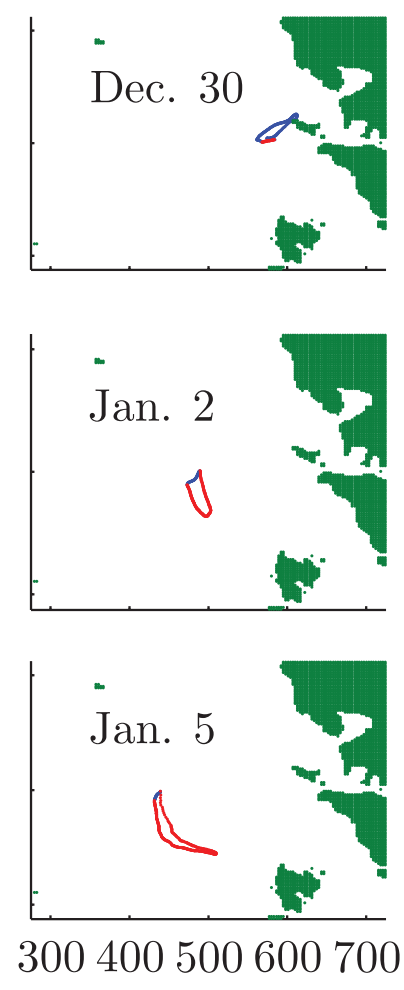

$x[\mathrm{~km}]$
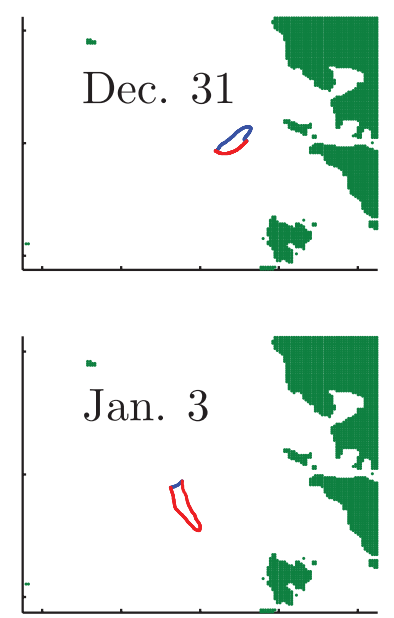

300400500600700

FIG. 8. Evolution of the lobe $L$ identified in Fig. 7.

considered in this section but may be typical of windgenerated dipoles in this region.

For pictures such as Fig. 7 to be useful, hyperbolic trajectories and attendant stable and unstable manifolds must exist for a sufficiently long time interval. The typical lifetime of the dipoles in this region is 2-4 weeks, and this period should be sufficiently long for a manifold to wrap around the edges of the gyres and form multiple lobes. Breakup of the eddy means that the geometrical template formed by the LCSs is completely changed and that the original hyperbolic trajectory may become indistinguishable from neighboring trajectories. This requirement is closely related to a more formal criterion (e.g., Poje and Haller 1999) for the identification of stable and unstable manifolds in finite time, namely that the Eulerian time scale of the feature in question be much greater than the Lagrangian time scale. If we take the former to be the 2-4-week period over which the dipole remains coherent and the latter to be the 2-4-day winding time of a parcel around the edge of one of the gyres, then a reasonable scale separation exists.

\section{b. Pathways into the dipole and biology}

Water that is swept westward through the axis of the dipole and around its edges carries physical and biological properties. These properties may be transported farther westward once the dipole breaks free of the coast. The possibility of biological transport is suggested by a satellite chlorophyll image (Fig. 10) indicating elevated levels associated with the cyclone and anticyclone (P08). The origins of this chlorophyll cannot be completely determined by this study, but we draw the reader's attention to the wrapping of elevated levels around the edge of the northern eddy. The manifolds suggest that water enters the dipole from the east, near the mouth of VIP. If a cluster of surface drifters is distributed over this area (blue circle in Fig. 11b) and evolved backward in time for 9 days (Fig. 11a), it can be seen that much of this water is stripped away from the coastline and from chlorophyll-rich Manila Bay slightly to the north. This water eventually becomes wrapped around the edges of the two eddies (Fig. 11c) in qualitative agreement with Fig. 10 (although this effect is much more pronounced for the northern eddy than for the southern eddy in Fig. 10). Note also that water contained near the cores of the two eddies (black and green patches in Fig. 11) did not come from chlorophyll-rich areas.

The export of properties from Manila Bay is further demonstrated by tracking tagged fluid parcels that leave the bay with an elevated chlorophyll level. Over the 

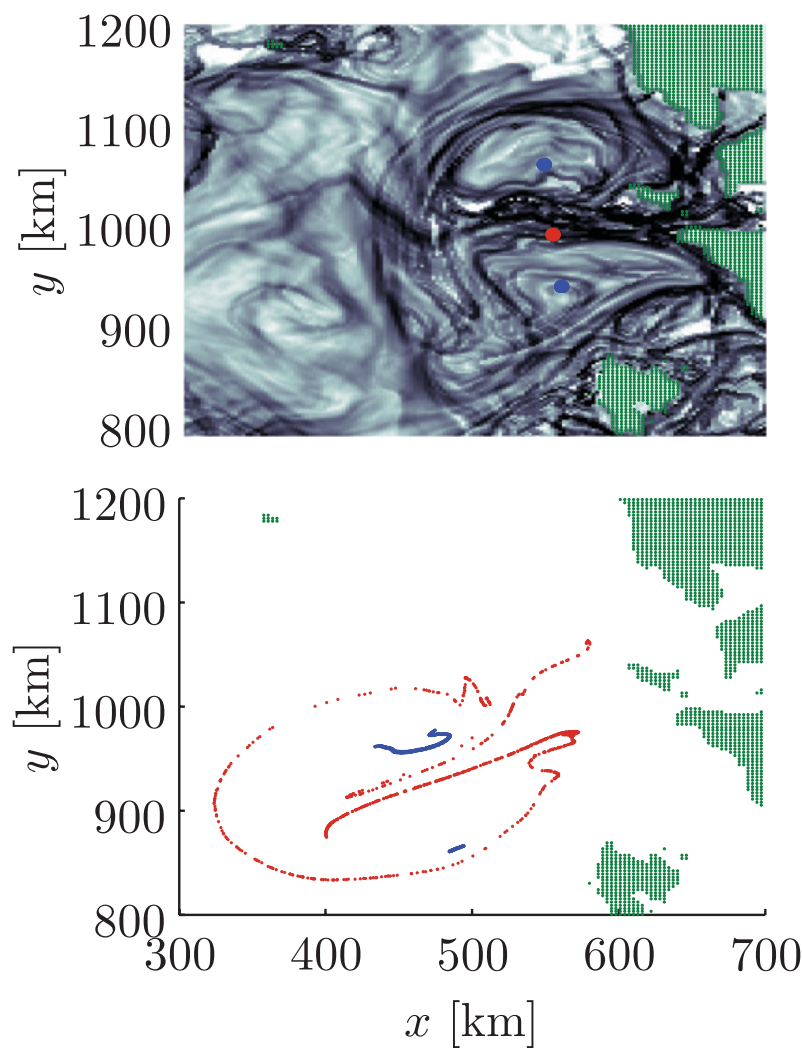

FIG. 9. Evolution over 10 days of patches of surface drifters launched in the eddy cores (blue) and in a region densely covered by intersecting manifolds (red). (top) Manifolds and drifter patches on 3 Jan 2005. (bottom) The drifter patches 10 days later.

period 25 December-13 January, 2550 parcels were released, once per day, from the bay and immediately surrounding waters. Parcels originating from the bay were initialized with an elevated nutrient level $N_{\text {release }}=$ $15 \mathrm{mmol} \mathrm{N} \mathrm{m}{ }^{-3}$, whereas parcels just outside the bay were given the initial value $N_{\text {release }}=0.05 \mathrm{mmol} \mathrm{N} \mathrm{m}^{-3}$. Each parcel was tracked and a simplified biological (NP) model (appendix A) was integrated along the parcel trajectory. The result of this calculation (Fig. 12) is a train of surface parcels that stream out of the bay and find their way into the edges of the eddies, with slightly higher parcel densities around the northern anticyclone. The color-coded chlorophyll values for the parcels are overestimated by the model, perhaps because zooplankton interactions and processes such as upwellingdownwelling and iron sequestration are ignored, but Manila Bay is clearly established as a potential source region. This also has implications for pollutant transport of coastal and Manila Bay localized sources.

\section{c. Filamentation and mixing}

The distinction between stirring and mixing can be described in terms of balances arising in the advection-diffusion equation for the concentration $C$ of a passive tracer,

$$
\frac{\partial C}{\partial t}+\mathbf{u} \cdot \nabla C=\nabla \cdot(\kappa \nabla C)
$$

For the present, we will restrict attention to 2D flows, and thus $\kappa$ should be interpreted as a horizontal eddy diffusivity that parameterizes the effects of any unresolved turbulence, here existing at or below the submesoscale. Stirring is a conservative process that is imagined to occur in an environment where $\kappa$ is sufficiently small and $C$ is sufficiently smooth that a balance exists between the two left-hand terms. The right-hand term may act over very long time scales but is inconsequential over the typical turnover times of the resolved eddies. However, even though $C$ may be initially smooth or may be introduced smoothly at large scales, the process of exponential stretching and folding amplifies the gradients of $C$ and creates more structure and smaller scales. At some small scale, the diffusion term becomes as large as the advection term and the evolution of the tracer field can no longer be described as conservative stirring, by any definition.

It is tempting to identify a length scale at which the advection and diffusion terms in Eq. (2) become comparable and to think of this length as the scale at which stirring leads to rapid mixing. In a region inhabited by Lagrangian chaos, this idea can be quantified through consideration of the evolution of a small disk of fluid (Fig. 13). The disk has initial radius $d_{0}$ and is stretched into a filament with constant area, with exponentially growing length $l$ and exponentially decaying width $w$ :

$$
l=d_{0} e^{\lambda t} \quad \text { and } \quad w=d_{0} e^{-\lambda t},
$$

where $\lambda$ is the stretching rate, which is here approximated by the finite-time Lyapunov exponent. For a constant $\kappa$ a simple scale analysis of Eq. (2) suggests that the advective and diffusive terms come into balance when the filament width shrinks to

$$
w_{a} \propto\left(\frac{\kappa}{\lambda}\right)^{1 / 2} .
$$

A similar result was derived by Garrett (1983) based on the rms strain rate of mesoscale eddies $\gamma$, in place of $\lambda$. In either case, the scale $w_{a}$ can be thought of as an arrest scale below which filaments cannot exist. Of course, the process parameterized by $\kappa$ may, in fact, consist of stretching, folding, and filamentation at unresolved scales. In this case, the idea of an arrest scale may be valid only at scales associated with the molecular diffusivity. 


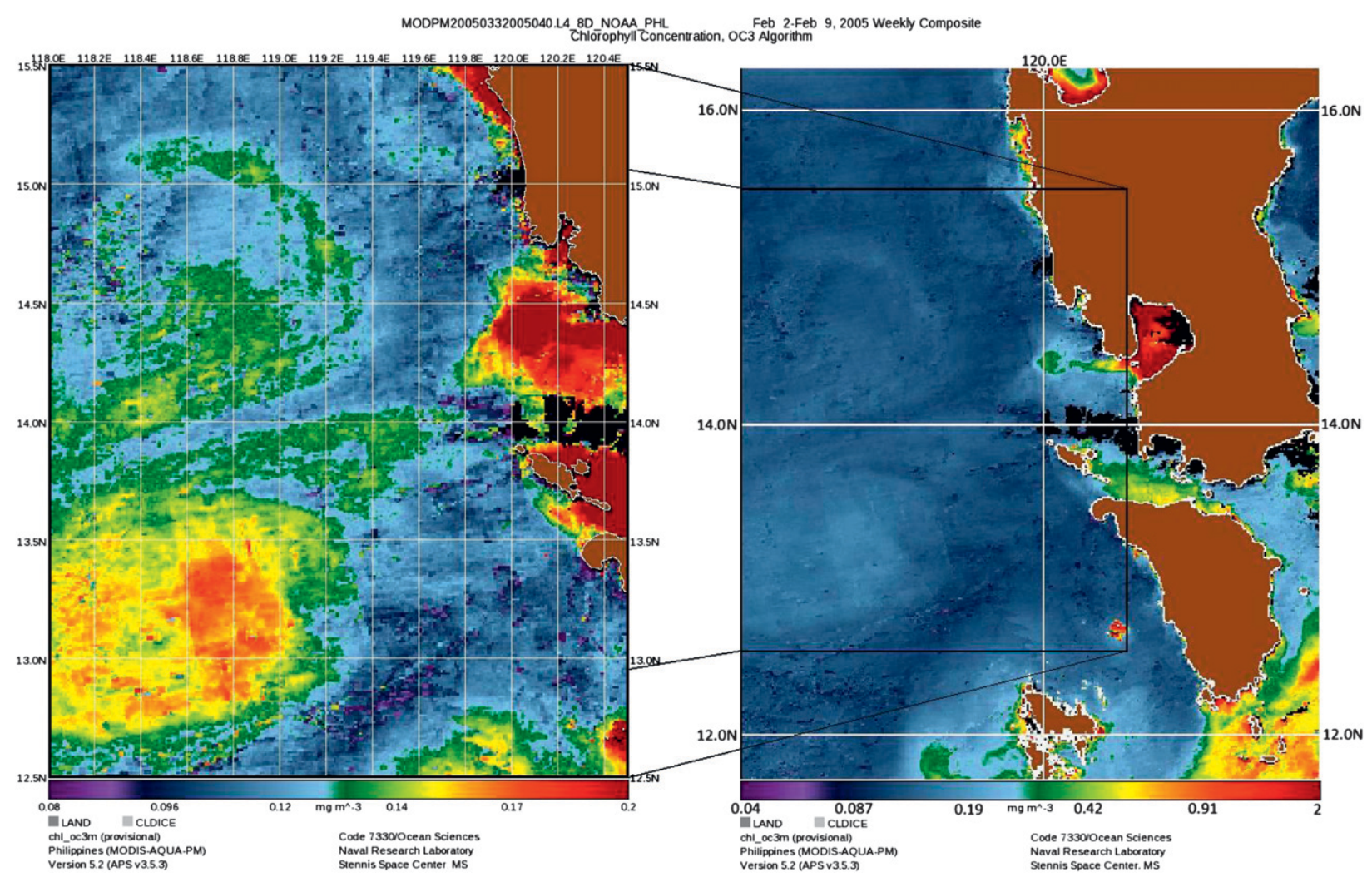

FIG. 10. Weekly composite chlorophyll image from beginning of February 2005: (left) Courtesy of Robert Arnone and (right) from Pullen et al. (2008).

To pursue this idea a bit further, assume that chaotic advection is indeed a valid model for stirring below the mesoscale. Then by definition $\lambda$ should be set by the mesoscale eddies and imposed at smaller scales. Because the eddies are resolved by our numerical models, we will use a value $\lambda \cong 1 \mathrm{day}^{-1}$ characteristic of the regions of more rapid stirring. Estimation of $\kappa$ is more problematic: a naive guess would be to use values $\kappa=$ $1-10 \mathrm{~m}^{2} \mathrm{~s}^{-1}$, based on observations of horizontal dispersion at the ocean surface by Okubo (1971) for the smallest scales (a few kilometers) resolved in our model. The resulting $w_{a}$ lies between $300 \mathrm{~m}$ and $1 \mathrm{~km}$.

A more careful approach acknowledges that Okubo's $\kappa$ is scale dependent. His fit to data based on horizontal scales $l$ ranging from $100 \mathrm{~m}$ to $100 \mathrm{~km}$ is

$$
\kappa=0.0103 l^{1.15},
$$

where $\kappa$ is expressed in $\mathrm{cm}^{2} \mathrm{~s}, l$ is in centimeters, and the constant 0.0103 has the units $\mathrm{cm}^{0.85} \mathrm{~s}^{-1}$. Because the diffusivity appropriate to Eq. (4) is the diffusivity at the arrest scale, we use (5) to substitute for $\kappa$ in Eq. (4) and set $l=w_{a}$,

$$
w_{a}=\sqrt{\frac{0.0103 w_{a}^{1.15}}{\lambda}} .
$$

Setting $\lambda=1$ day $^{-1}$ and solving for $w_{a}$ gives

$$
w_{a} \cong 30 \mathrm{~m} .
$$

(Okubo also notes that a local fit to data over the range $40 \mathrm{~m}-1 \mathrm{~km}$ is closer to $\kappa=c l^{4 / 3}$, where we estimate $c=$ $0.0022 \mathrm{~cm}^{2 / 3} \mathrm{~s}^{-1}$ based on his Fig. 4. Using this expression instead of Eq. (5) leads to a value for $w_{a}$ of $28 \mathrm{~m}$.)

The estimate $w_{a} \cong 30 \mathrm{~m}$ is well below the "naïve" estimate listed earlier and is just slightly below the scale of measurements on which Eq. (5) is based. Processes such as internal waves may come into play at such small scales, and it is dubious that $\lambda=1 \mathrm{day}^{-1}$ continues to represent the stretching rate. Higher stretching rates would tend to reduce the estimate of $w_{a}$ further.

The calculation of $w_{a}$ clearly involves a number of assumptions that may or may not be valid. An arrest scale has been identified in other settings, one being Smith and Ferrari's (2009) study of three-dimensional, quasigeostrophic thermocline turbulence. We next examine 

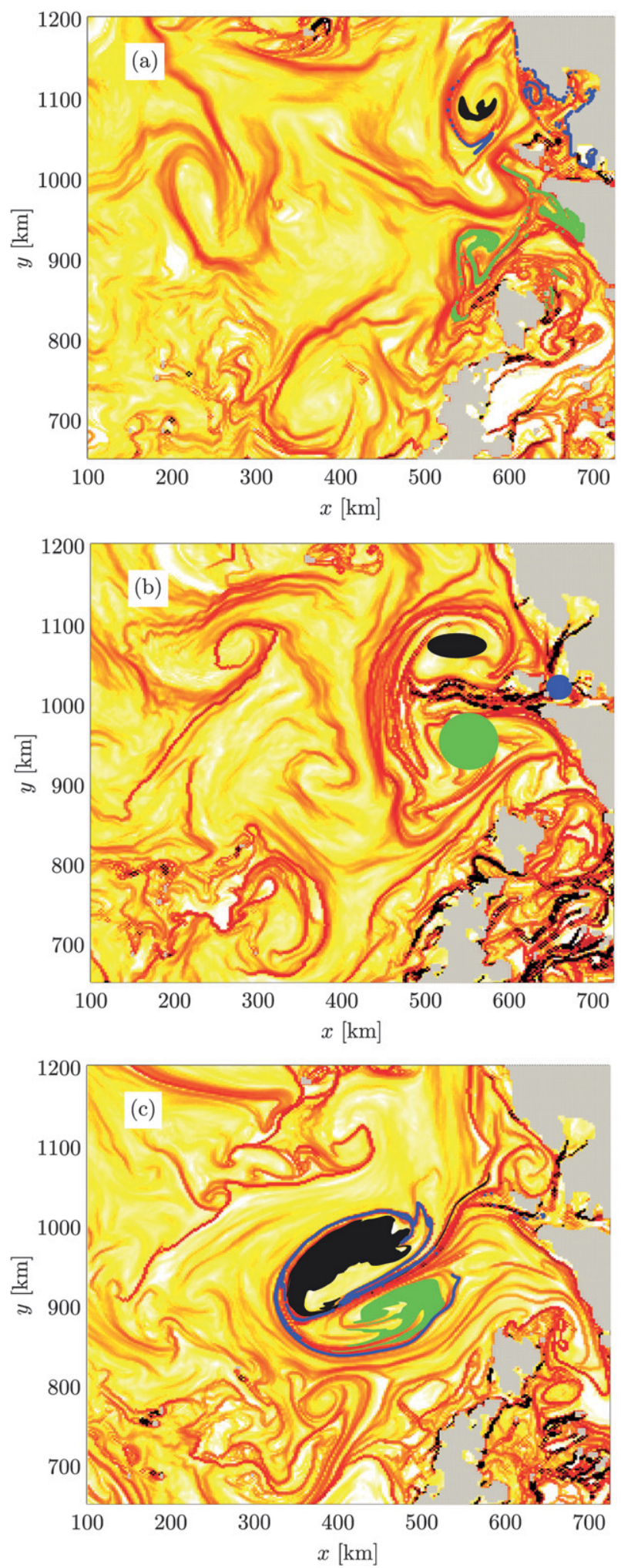

FIG. 11. Evolution over (a) 9 days backward and (c) 10 days forward in time from 3 Jan of (b) concentrated patches of surface drifters launched in the cores of the eddies (black and green) and over a region near the mouth of VIP (blue). Background shows (a) FTLEf and (b),(c) FTLE-b.

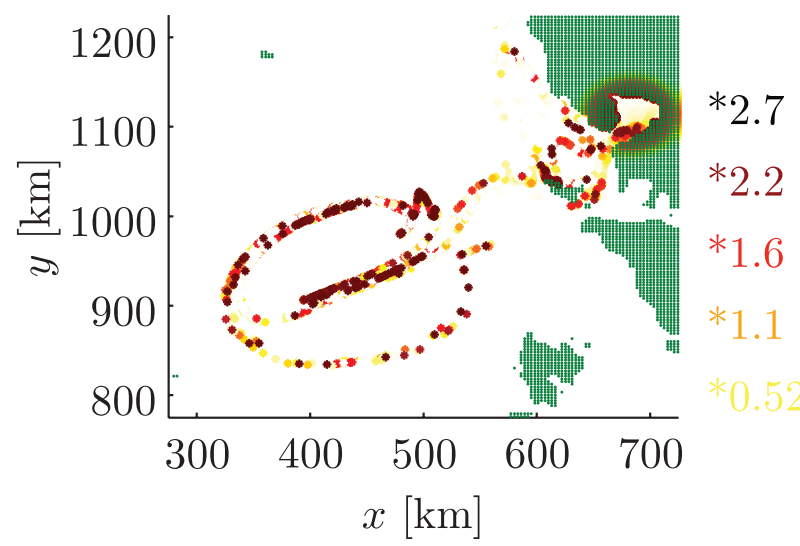

FIG. 12. Simulated chlorophyll concentration $\left(\mathrm{mg} \mathrm{Chl} \mathrm{m}^{-3}\right)$ for 13 Jan 2005 computed by solving an NP biological model (explained in appendix A) along characteristics (particle trajectories) of the flow.

a near-surface, underway temperature record from a dipole to seek evidence of filament arrest.

\section{d. Analysis of an underway temperature record}

Evidence of dipoles and filaments west of Panay and Mindoro Islands are apparent in the PHILEX Regional Intensive Observing Period 2008 (RIOP08) January data (Fig. 14). The near-surface velocity from the shipboard ADCP (Fig. 14a) reveals a well formed cyclonic eddy between $12^{\circ}$ and $13.5^{\circ} \mathrm{N}$ and $119^{\circ}$ and $121^{\circ} \mathrm{E}$. The near-surface temperature along a north-south track through the cyclone (Fig. 14b) shows strong fluctuations of $0.2^{\circ}-0.4^{\circ} \mathrm{C}$ that may be associated with cold and warm filaments of surface water. The southern segment of the track crosses the southern edge of the cyclone, a region where we expect such filaments, and indeed the temperature variance is elevated over this segment.

The spectrum of horizontal temperature variance (Fig. 14c) shows a gradual decay, becoming less steep at higher wavenumber, and no abrupt roll off that might indicate the presence of an arrest scale. However, the $95 \%$ confidence limits indicate that the shape of the spectrum is quite uncertain in the wavenumber range close to the predicted $w_{a} \cong 30 \mathrm{~m}$. In summary, the underway temperature section shows the presence of filaments near the edge of the dipole, where expected, but is too short to permit firm conclusions regarding the presence of an arrest scale.

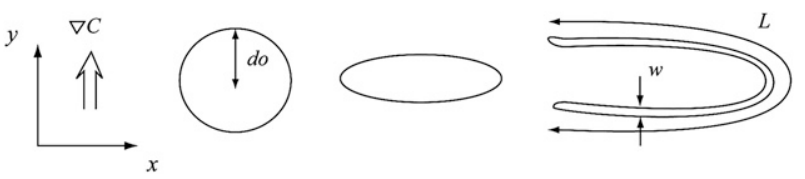

FIG. 13. The stretching and folding of a blob of passive tracer in a $2 \mathrm{D}$ horizontal flow field. 


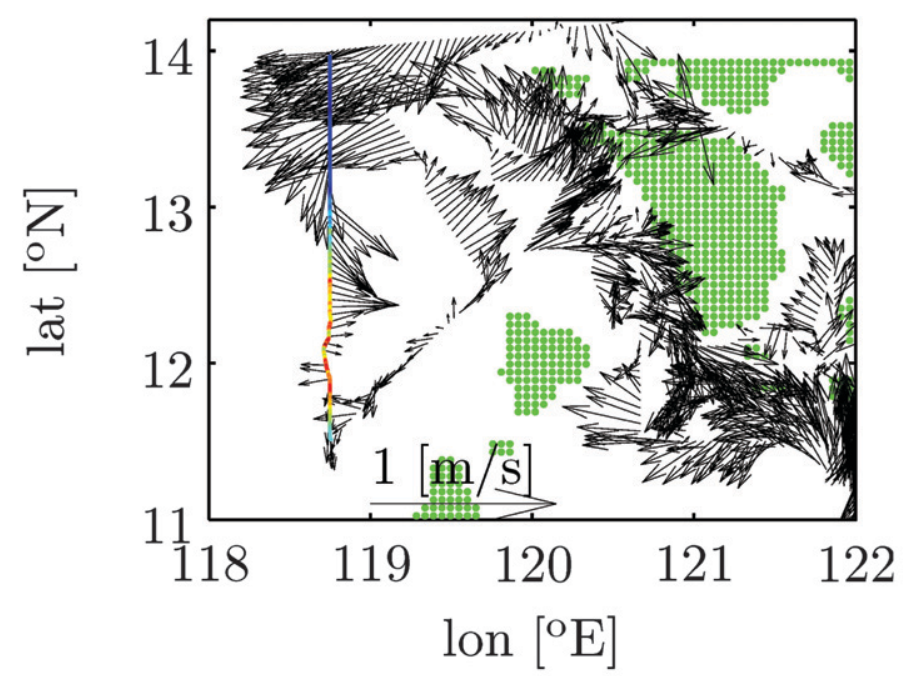

(a)

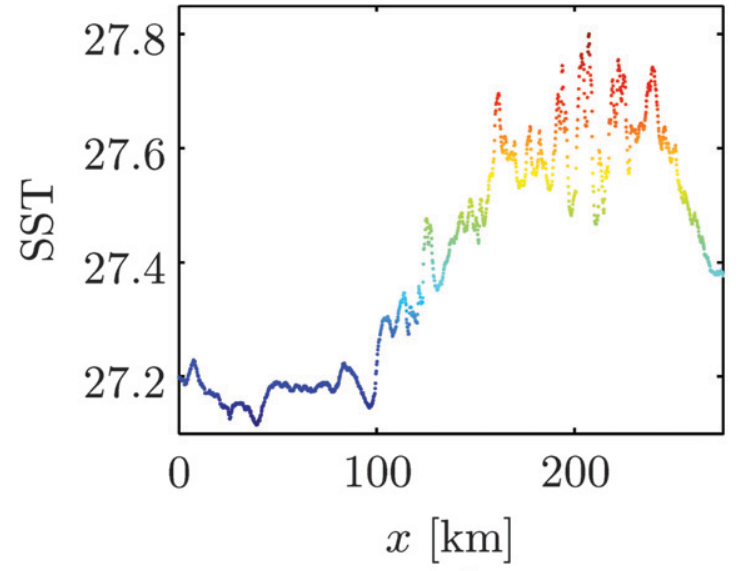

(b)

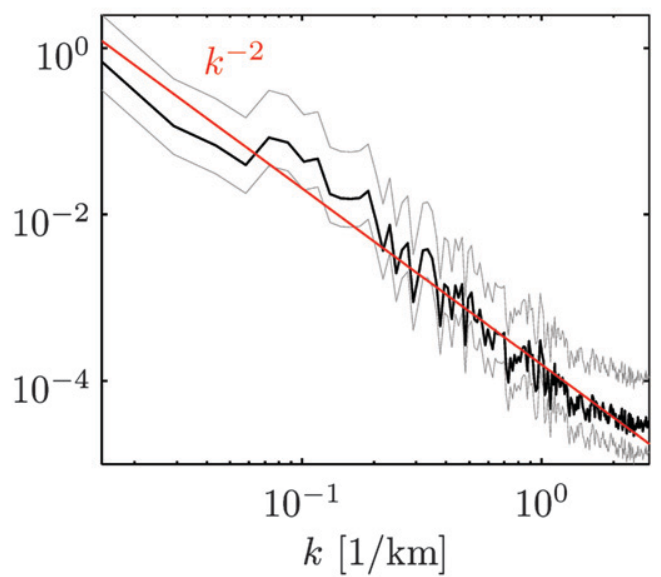

(c)

FIG. 14. (a) Near-surface (25-55 m average) velocity from the shipboard ADCP, January 2008, from the PHILEX RIOP08 cruise. The cyclone is evident between $12^{\circ}$ and $13.5^{\circ} \mathrm{N}$ and between $119^{\circ}$ and $121^{\circ} \mathrm{E}$. (b) Near-surface temperature along the ship track at $118.75^{\circ} \mathrm{E}$, from $14^{\circ} \mathrm{E}(x=0)$ to $11.5^{\circ} \mathrm{N}(x=275 \mathrm{~km})$, also shown in (a). (c) Horizontal wavenumber spectrum for near-surface temperature shown in (b); gray lines indicate a $95 \%$ confidence interval.

\section{Summary and conclusions}

We have explored the relevance of chaotic advection and the utility of dynamical systems methodology in the elucidation of near-surface horizontal stirring processes in an archipelago. Motivation stems from the idea that the natural geometry of a system of semienclosed basins and channels should give rise to the long-lived eddies, gyres, and boundary separation phenomena that produce clearly identifiable Lagrangian coherent structures. Because the data requirements are daunting, we have relied on numerical simulations in place of observations to produce the surface velocity fields required for the dynamical systems analysis. Plots of Lagrangian coherent structures (that serve as proxy finite-time stable and unstable manifolds) over the whole Philippine region reveal features that bear resemblance to textbook examples, including the pairs of like-signed eddies (the Duffing oscillator), opposite-signed eddies (dipoles), boundary-trapped eddies, etc. Closer inspection reveals additional complexity, including the presence of multiple hyperbolic trajectories and manifolds in close proximity. Nevertheless, the manifolds are robust and visually smooth on the scale of the mesoscale eddies and gyres suggesting that stirring is dominated by advection due to the eddies. Maps of manifolds over large scales reveal the presence of weakly stirred regions imbedded in strongly stirred ones. This dichotomy is verified by releases of 
tracers or marked parcels in the two regions. All of these features are hallmarks of chaotic advection and the suggestion is that this process is relevant over scales down to $\sim 5-\mathrm{km}$ resolution of the models.

When focused on a particular feature, in our case a wind-generated dipole, dynamical systems methodology reveals the clear presence of turnstile lobes. Although the manifolds and lobes are more complex and irregular than what might be encountered in a textbook, they provide a clear description of a process by which surface water is carried though the dipole axis, exchanged between the two gyres, and stretched into filaments that are wrapped around the edges of the eddies. The implications for transport of biological properties are discussed; transport of chlorophyll by a wind-driven dipole is modeled and results are compared with satellitemeasured estimates of chlorophyll concentration in the area. Both our simulations and satellite measurements indicate elevated levels of chlorophyll near the perimeter of the dipole (although satellite measurements indicate that this effect is more pronounced for the northern gyre of the dipole). A potential source of this chlorophyll is shown to be located in Manila Bay.

Chaotic advection provides an effective mechanism for creating fine structure from a smooth large-scale tracer distribution. This mechanism, which is known as filamentation, involves the stretching of blobs of tracer into elongated filaments whose widths decrease at an exponential rate. Measured near-surface temperature shows evidence of filamentation where we expect (i.e., near the edge of the dipole). In principle, the process is halted when the filament width reaches an arrest scale at which further narrowing is balanced by the spreading tendency associated with nonconservative material exchange at small scales, here parameterized by a submesoscale diffusivity.

Our estimate of the arrest scale $(\cong 30 \mathrm{~m})$ is based on a number of assumptions, including the use of a fixed stretching rate $\lambda$ set at the mesoscale and of Okubo's (1971) scale-dependent horizontal diffusivity. A factor missing from our discussion is the three-dimensional structure of filaments and, in particular, the tilting of the filaments by vertical shear (some of the effects of horizontal dispersion due to vertical shear are likely present in Okubo's diffusivities because these are based on dye release experiments). Smith and Ferrari (2009) considered this effect in their study of quasigeostrophic turbulence in the thermocline. In their conceptual model, the narrowing of a filament in the direction normal to a constant tracer surface is halted through the effects of an isotropic diffusivity, perhaps because of breaking internal waves. To predict the arrest scale, they invoke the argument of Charney (1971) that the filament slope tends toward a limited value $f / N$ to conserve quasigeostrophic potential vorticity. They show that the kinetic energy spectrum, plotted in terms of the scaled total wavenumber $\mu=\left(k^{2}+l^{2}+f^{2} m^{2} / N^{2}\right)^{1 / 2}$, does indeed roll off for $\mu$ values higher than the predicted arrest scale. A comparison with our $w_{a}$ could be made in terms of the horizontal projection of their arrest scale,

$$
w_{a} \sim \frac{N}{f}\left(\frac{\kappa_{\mathrm{isotr}}}{\lambda}\right)^{1 / 2} .
$$

However, it is quite difficult to know what stretching rate $\lambda$ should be used for the three-dimensional problem. In addition, the notion of conservation of quasigeostrophic potential vorticity is dubious for the upper ocean, so we have pursued this no further.

Acknowledgments. This work was supported by the Office of Naval Research: Grant N00014-07-1-0590 to Woods Hole Oceanographic Institution; Grant ONR0601153N to Stevens Institute of Technology; Grant N00014-07-1-0417 to Institute of Marine and Coastal Sciences, Rutgers University; and Grant N00014-09-10582 to Lamont-Doherty Earth Observatory of Columbia University. We thank the reviewers for a number of helpful suggestions.

\section{APPENDIX A}

\section{Biological Model}

The nutrient-phytoplankton (NP) model used in section 5 was

$$
\frac{d N}{d t}=-\mu \frac{N}{N+N_{0}} P+\epsilon \sigma P, \quad \frac{d P}{d t}=\mu \frac{N}{N+N_{0}} P-\sigma P .
$$

The coefficient values for the Philippine region are difficult to constrain, so we used typical values (e.g., Olascoaga et al. 2008). They include the maximum rate of nutrient-limited phytoplankton growth $\mu=0.4$ day $^{-1}$, the half-saturation constant $N_{0}=0.5\left(\mathrm{mmol} \mathrm{N} \mathrm{m}^{-3}\right)$, the portion of recyclable nutrients in dead phytoplankton $\epsilon=0.1$, and the linear phytoplankton loss $\sigma=$ 0.1 day $^{-1}$. In addition, the initial condition for phytoplankton $P$ is $0.01\left(\mathrm{mg} \mathrm{Chl} \mathrm{m}^{-3}\right)$ everywhere.

\section{APPENDIX B}

\section{The Basic Principles of the Individual Trajectory Method (ITM)}

The correlation dimension $C$ of a trajectory is a measure of the area in $(x, y)$ occupied by a trajectory. The 
correlation dimension can be effectively estimated using a box counting algorithm (Brown 1998). To implement this algorithm, the domain of interest is mapped onto the unit square, which is then divided into $2^{2 m}, m=0,1, \ldots$, $m_{\max }$ boxes. The area of each box is $s^{2}=2^{-2 m}$. For each $m$, probabilities $P_{j}, j=1, \ldots, 2^{2 m}$ are computed that the trajectory visits the $j$ th box. After that, the distribution function $F(s)=\sum_{j}\left[P_{j}(s)\right]^{2}$ is constructed and the scaling relationship between the area of the box $s$ and the distribution function $F(s)$,

$$
F(s) \simeq s^{C},
$$

is used to estimate the correlation dimension $C$. In 2D flows, $C$ can vary from 0 (for a stationary point of a flow) to 1 (for a smooth $1 \mathrm{D}$ curve) to 2 (for a chaotic curve that densely covers a $2 \mathrm{D}$ area). The anticipated connection between trajectory complexity (as measured by $C$ ) and stable and unstable manifolds can now be stated.

Trajectories lying on a stable manifold will rapidly approach the hyperbolic trajectory. If the hyperbolic trajectory remains confined to a boundary or a small area of the flow field, then the $C$ value for a trajectory on a stable manifold will be smaller than that of the unconstrained, neighboring motion. Also, because trajectories on opposite sides of a stable manifold are generally headed toward different regions after they pass the hyperbolic trajectory, their $C$ values are expected to be qualitatively different. A similar statement clearly holds in backward time for trajectories lying on an unstable manifold. With this in mind, stable/unstable manifolds correspond to minimizing ridges and curves of highest gradient of the $C$ field computed in forward/ backward time. ITMs appear to have some advantages in terms of resolution over FTLEs, but the theoretical underpinnings are not well established and there are some subtleties in interpretation. Indeed, one of the objectives of this study is to compare the two methods in the setting of a complex flow with realistic mesoscale features. For the purposes of estimating the rough positions of manifolds, we have found that ITM results are equivalent to those based on FTLEs.

\section{REFERENCES}

Bennett, A. F., 1984: Relative dispersion: Local and nonlocal dynamics. J. Atmos. Sci., 41, 1881-1886.

Brown, M. G., 1998: Phase space structure and fractal trajectories in $1 \frac{1}{2} 2$ degree of freedom Hamiltonian systems whose time dependence is quasiperiodic. Nonlinear Processes Geophys., 5, $69-74$.

Charney, J. G., 1971: Geostrophic turbulence. J. Atmos. Sci., 28, 1087-1095.

Egbert, G., and L. Erofeeva, 2002: Efficient inverse modeling of barotropic ocean tides. J. Atmos. Oceanic Technol., 19, 183-204.
Fairall, C. W., E. F. Bradley, J. E. Hare, A. A. Grachev, and J. B. Edson, 2003: Bulk parameterization of air-sea fluxes: Updates and verification for the COARE algorithm. J. Climate, 16, 571-591.

Flather, R. A., 1976: A tidal model of the northwest European continental shelf. Mem. Soc. Roy. Sci. Liege, 6, 141-164.

Garrett, C., 1983: On the initial streakiness of a dispersing tracer in two- and three-dimensional turbulence. Dyn. Atmos. Oceans, 7, 265-277.

Haller, G., 2001a: Distinguished material surfaces and coherent structures in 3D fluid flows. Physica D, 149, 248-277.

- 2001b: Lagrangian structures and the rate of strain in a partition of two-dimensional turbulence. Phys. Fluids, 13, 3365-3385.

_ 2002: Lagrangian coherent structures from approximate velocity data. Phys. Fluids, 14, 1851-1861.

_ 2005: An objective definition of a vortex. J. Fluid Mech., 525, $1-26$.

Koh, T. Y., and R. A. Plumb, 2000: Lobe dynamics applied to barotropic Rossby wave breaking. Phys. Fluids, 12, 1518-1528. , and B. Legras, 2002: Hyperbolic lines and the stratospheric polar vortex. Chaos, 12, 328-394.

Kuznetsov, L., M. Toner, A. D. Kirwan, C. K. R. T. Jones, L. H. Kantha, and J. Choi, 2002: The Loop Current and adjacent rings delineated by Lagrangian analysis of the nearsurface flow. J. Mar. Res., 60, 405-429.

Lukovich, J. V., and T. G. Shepherd, 2005: Stirring and mixing in two-dimensional divergent flow. J. Atmos. Sci., 64, 3933-3954.

Mancho, A. M., D. Small, S. Wiggins, and K. Ide, 2003: Computation of stable and unstable manifolds of hyperbolic trajectories in two-dimensional, aperiodically time-dependent vector fields. Physica D, 182, 188-222.

,-- , and,- 2004 : Computation of hyperbolic trajectories and their stable and unstable manifolds for oceanographic flows represented as data sets. Nonlinear Processes Geophys., 11, 17-33.

,-- , and -2006 : A tutorial on dynamical systems concepts applied to Lagrangian transport in oceanic flows defined as finite time data sets: Theoretical and computational issues. Phys. Rep., 437, 55-124.

Miller, P. D., C. K. R. T. Jones, A. M. Rogerson, and L. J. Pratt, 1997: Quantifying transport in numerically generated velocity fields. Physica D, 110, 105-122.

— L. J. Pratt, K. R. Helrich, and C. K. R. T. Jones, 2002: Chaotic transport of mass and potential vorticity for an island recirculation. J. Phys. Oceanogr., 32, 80-102.

Mizuta, R., and S. Yoden, 2001: Chaotic mixing and transport barriers in an idealized stratospheric polar vortex. J. Atmos. Sci., 58, 2616-2629.

Moore, A. M., H. G. Arango, E. Di Lorenzo, B. D. Cornuelle, A. J. Miller, and D. J. Neilson, 2004: A comprehensive ocean prediction and analysis system based on the tangent linear and adjoint of a regional ocean model. Ocean Modell., 7, 227-258.

Ngan, K., and T. G. Shepherd, 1999a: A closer look at chaotic advection in the stratosphere. Part I: Geometric structure. J. Atmos. Sci., 56, 4134-4152.

— and 1999b: A closer look at chaotic advection in the stratosphere. Part II: Statistical diagnostics. J. Atmos. Sci., 56, 4153-4166.

Okubo, A., 1971: Oceanic diffusion diagram. Deep-Sea Res., 18, 789-802.

Olascoaga, M. J., F. J. Beron-Vera, L. E. Brand, and H. Koçak, 2008: Tracing the early development of harmful algal blooms 
on the West Florida Shelf with the aid of Lagrangian Coherent Structures. J. Geophys. Res., 113, C12014, doi:10.1029/ 2007JC004533.

Plumb, R. A., 2002: Stratospheric transport. J. Meteor. Soc. Japan, 80, 793-809.

Poje, A. C., and G. Haller, 1999: Geometry of cross-stream mixing in a double-gyre ocean model. J. Phys. Oceanogr., 29, 16491665.

Pullen, J., J. D. Doyle, P. May, C. Chavanne, P. Flament, and R. A. Arnone, 2008: Monsoon surges trigger oceanic eddy formation and propagation in the lee of the Philippine Islands. Geophys. Res. Lett., 35, L07604, doi:10.1029/2007GL033109.

Rogerson, A., P. D. Miller, L. J. Pratt, and C. K. R. T. Jones, 1999: Lagrangian motion and fluid exchange in a barotropic meandering jet. J. Phys. Oceanogr., 29, 2635-2655.

Rypina, I. I., M. G. Brown, F. J. Beron-Vera, H. Kocak, M. J. Olascoaga, and I. A. Udovydchenkov, 2007: On the Lagrangian dynamics of atmospheric zonal jets and the permeability of the stratospheric polar vortex. J. Atmos. Sci., 64, 3593-3610.

$\longrightarrow,-$, and H. Kocak, 2009: Transport in an idealized threegyre system with an application to the Adriatic Sea. J. Phys. Oceanogr., 39, 675-690.

Samelson, R. M., 1992: Fluid exchange across a meandering jet. J. Phys. Oceanogr., 22, 431-440.
, and S. Wiggins, 2006: Lagrangian Transport in Geophysical Jets and Waves: The Dynamical Systems Approach. SpringerVerlag, $147 \mathrm{pp}$

Shadden, S. C., F. Lekien, and J. E. Marsden, 2005: Definition and properties of Lagrangian coherent structures from finite-time Lyapunov exponents in two-dimensional aperiodic flows Physica D, 212, 271-304.

Shchepetkin, A. F., and J. C. McWilliams, 2005: The regional ocean modeling system: A split-explicit, free-surface, topography following coordinates ocean model. Ocean Modell., 9, 347-404.

Shepherd, T. G., J. N. Koshyk, and K. Ngan, 2000: On the nature of large-scale mixing in the stratosphere and mesosphere. J. Geophys. Res., 105, 12 433-12 446.

Smith, K. S., and R. Ferrari, 2009: The production and dissipation of compensated thermocline variance by mesoscale stirring. J. Phys. Oceanogr., 39, 2477-2501.

Smith, W. H. F., and D. T. Sandwell, 1997: Global sea floor topography from satellite altimetry and ship depth soundings. Science, 277, 1956-1962.

Sommeria, J., S. D. Meyers, and H. L. Swinney, 1989: Laboratory model of a planetary eastward jet. Nature, 337, 58-61.

Tennekes, H., and J. L. Lumley, 1972: A First Course in Turbulence. MIT Press, 300 pp.

Wiggins, S., 2005: The dynamical systems approach to Lagrangian transport in oceanic flows. Annu. Rev. Fluid Mech., 37, 295-328. 